\title{
REVERSE-CURRENT CIRCUIT-BREAKERS AND THE PROTECTION OF TRANSMISSION LINES.
}

\author{
BY LEONARD WILSON.
}

Engineers who have had the experience of running faulty apparatus in parallel have long recognized the inadequacy of a system of excess-current protection; but the necessity for adopting reverse-current protective devices is still far from being universally recognized. The advantages of reverse-current protection over excess-current protection are briefly: That the former cuts out the faulty apparatus and does so at the moment when only a small current is flowing, thus causing the minimum disturbance to the system, while the latter is very liable to cut out all the healthy units instead of the one faulty one and only operates when the current is excessive. It will be seen, therefore, that wherever current is being fed to a common bus-bar from more than one source, reverse-current circuit-breakers should be installed.

The evolution of the reverse-current circuit-breaker has been a long process, and even at the present day devices are installed which do not possess the necessary characteristics for reliable operation and consequently bring discredit on this system of protection. The following are the more important of the characteristics referred to.

(1) A reverse current equal to 25 per cent. of the normal must be sufficient to operate the breakers.

(2) The magnetic pull must continue to increase with an increase of the reverse current, so that any sticking of the mechanical part will merely cause the breaker to operate at a sightly higher current, and that if the reverse current suddenly 
attains a very high value the operation of the breaker will be the more certain.

(3) A failure of the potential coil, from whatever cause arising, must not operate the breaker.

(4) The device must in some way continuously indicate whether it is in working order or not.

With regard to the first point, it is important that the device should not be too delicate in its action, and to obtain this, the relay is in some cases provided with a time-element. This, however, does not produce very satisfactory results as it allows the current (when there is a serious fault) to rise to too high a value The best practice seems to be to have quick-acting relays set for a moderate return current, 25 per cent, having been found a very

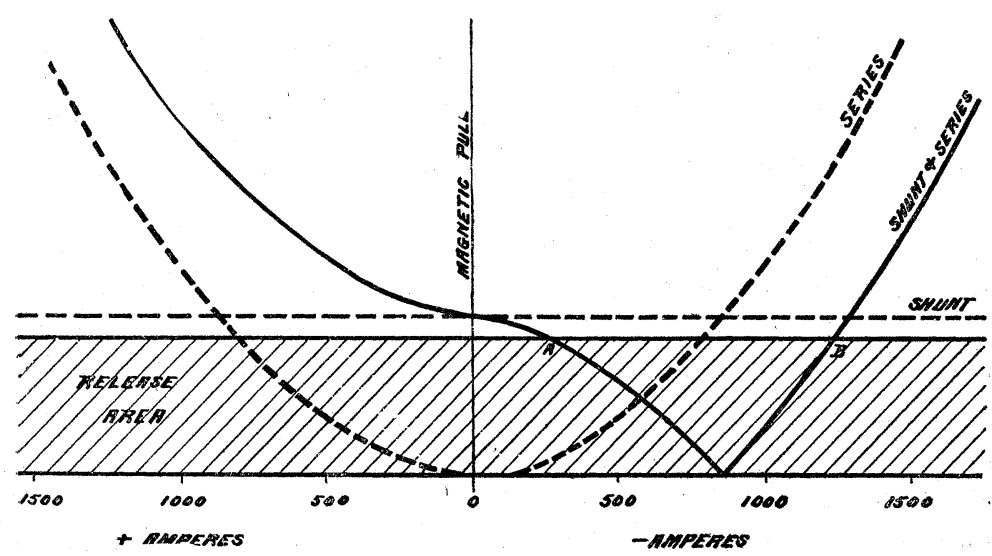

rof

suitable value. If for any reason, a combined reverse and excesscurrent breaker. is required it will be found better to provide two separate tripping-coils, as a combination of the two in one necessitates a certain compromise in the charactelistics of the device. A convenient method of illustrating the action of devices of this kind is that of plotting characteristic curves showing the relation between the operating force and the current producing it.

The force that will just operate the device is shown by the boundary of a shaded area, and the part of the curve lying outside this area indicates the range of the current at which the breaker remains closed. Fig. 1 shows the characteristic curves of a circuit-breaker which operates when the magnetic pull falls below 
a fixed value, and it will be seen that in this type the curve crosses the boundary of the shaded area at two points, $\mathrm{A}$ and $\mathrm{B}$. In consequence of this, if the reverse current suddenly attains a value higher than $\mathrm{E}$, the device will fail to operate. Also it will be noted that an interruption of the shunt current will operate the breaker. Fig. 2 shows the curve of a circuit breaker which operates when the magnetic pull, due to the product of the seires and shunt current, exceeds a certain value; and it will be seen that the objectionable features of No. 1 curve are absent from this.

It is unnecessary to describe in detail any particular form of reverse current circuit breaker, as such information is readily obtainable from the bulletins of manufacturing companies, but a

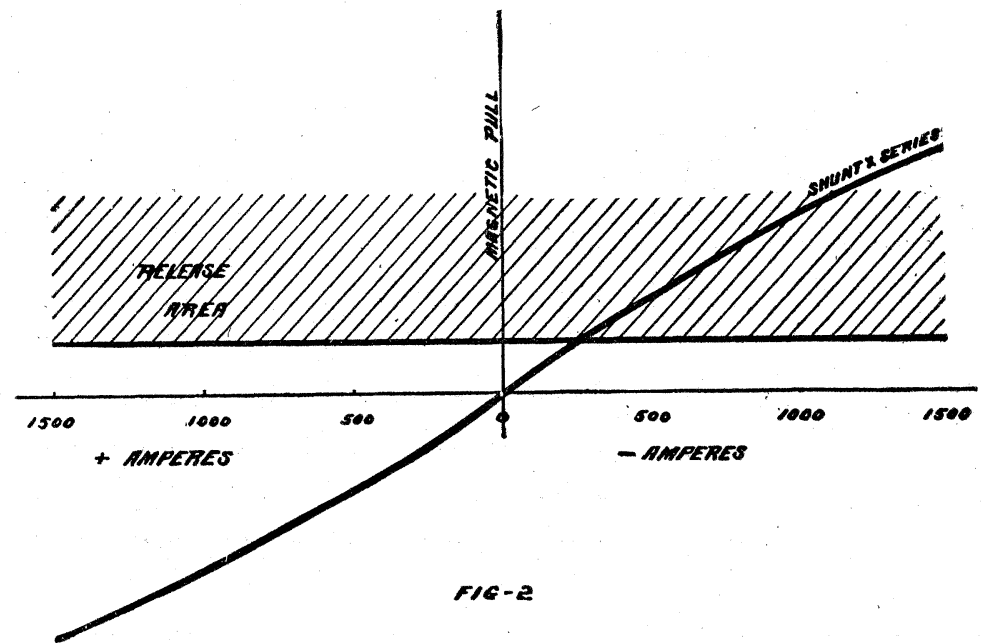

short description of a device, novel in this country, together with a demonstration of it in operation, may prove of interest.

Some operating engineers prefer to trust to the switchboard attendant in case of trouble rather than to install any fcrm of automatic circuit-breaker, but in such cases it is very necessary to have some visual signal to indicate which of a number of generators is the faulty one. For this purpose, some form of wattmeter relay arranged to clcse a local lamp circuit may be used; but a simpler and more reliable device is the one illustrated in Fig. 3. This, device, invented by Mr. L. Andrews, of England, consists of a double magnetic circuit magnetized by shunt and series windings and provided with two secondary windings connected to red and green signal lamps. In the diagram $\mathrm{A} \mathrm{A}$ is the 
shunt winding, connected across the station bus-bars and $\mathrm{B}$ is the series winding, consisting of cne turn of the main cable connecting the generator to the bus-bar. With only the shunt winding excited, the secondary coils c c have each half the normal lamp voltage induced in them, and consequently both red and green lamps glow faintly. When the generator delivers current to the bus-bar, the action of the series winding is to neutralize the flux through one secondary winding and increase it through the other, thus extinguishing the red lamp and lighting up the green one; conversely, when the current reverses, the green lamp goes out and the red one lights up. This device appeals to operating engineers by its simplicity and the complete absence of moving parts. The use of two lamps (preferably. each in duplicate)

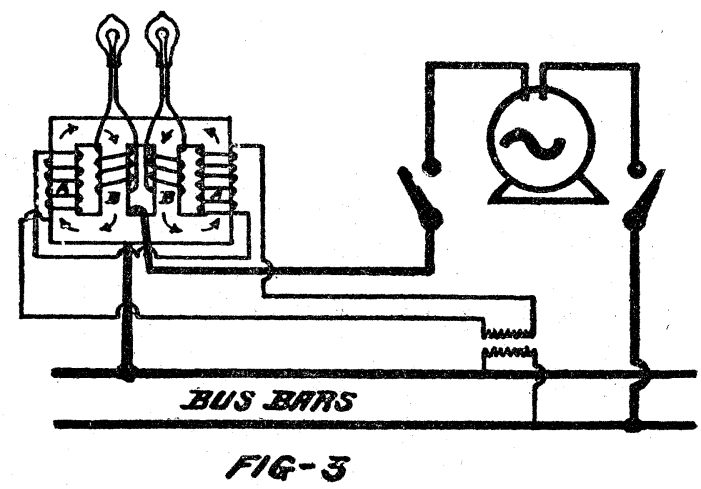

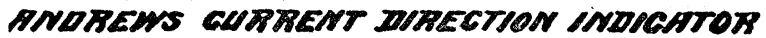

enables the switchboard attendant at any time to ascertain whether the devices are in working order, for whichever direction the current is flowing in and however small the current, it will prcduce a difference in glow, between the red and green lamps, which indicates that the device is operative.

The protecticn of the transmission lines presents many problems of interest. The simple case where the feeders starting frcm the bus-bars transmit power to separate receiving points is taken care of by excess-current circuit-breakers; but where the feeders are also connected together at the receiving end, some cther system of protection is necessary. An efficient system of protection for multiple transmission lines should, while normally allowing all the lines to be in use at once, be capable of preventing any excessive rush of current in case of a fault on one of the lines 
and should include devices for entirely cutting out such faulty line without serious disturbance of the rest of the system. Attempts have been made to provide such protection by the use of excess-current breakers at the generating end and reversecurrent breakers at the receiving end, but with very little success, the failures being due to one or both of the following defects in the system:

(1) That it does not prevent the sudden rush of current through the healthy mains to the faulty one.

(2) In the case of a low-resistance fault the pressure falls so low at the receiving end that the reverse-current-breakers (if they depend on the action of potential coils) fail to operate.

The system devised by Mr. L. Andrews, which is briefly referred to in another paper, includes a choking-roil which absolutely prevents any excessive rush of current in case of a fault on one of the mains, and this in combination with automatic cut-out devices: fulfils the necessary conditions for complete protection without. interruption of the supply. As used in England, this system has: only been applied to duplicate mains, and $f$ or this a single chokingcoil is used connected across the two mains, with a tap from the middle of the winding going to the receiving apparatus. By the use of more chokers the system can be adapted for any number of feeders in parallel. By the use of choking-coils connected in this manner, the principal difficulties usually encountered in the protection of multiple transmission lines are avoided; sudden current rushes are absolutely prevented; the voltage at the receiving end is maintained, and consequently the operation of the autcmatic devices can be relied on.

Fig. 4 represents a duplicate transmission line protected at the generator end by excess-current circuit-breakers and at the receiver end equipped with differential choke-coils. For the sake of simplicity, only one polarity of the system is shown thus equipped. When a short-circuit occurs on, say, в feeder, there is a big rush of current from the generator to the fault through $\mathrm{B}$, and this operates the excess-current circuit-breaker at the generator end. Without the choking-coil, there would also be a rush of current to the fault via the healthy feeder and this would very probably operate the remaining circuit-breaker and interrupt the supply. The choke-coil entirely prevents this, for as sjon as the current flow in the two halves becomes unbalanced. an e.m.f. is induced which opposes the unbalancing, and the only current which flows to the fault through the healthy feeder is that 
required to magnetize the choking-coil. While the short-circuit is maintained and before the faulty feeder is cut out, the receiver voltage will be 50 per cent. of its normal value. The switch $\mathrm{B}$, being thrown, the faulty feeder is cut out, the choking-coil shortcircuited and the supply is continued at normal voltage. In the apparatus exhibited, a special form of automatic device is used for operating the feeder switches and this device is illustrated diagrammatically in Fig. 5. The two small transformers are each magnetized by two windings connected across the system sn that the induced flux of one of them depends on the voltage of

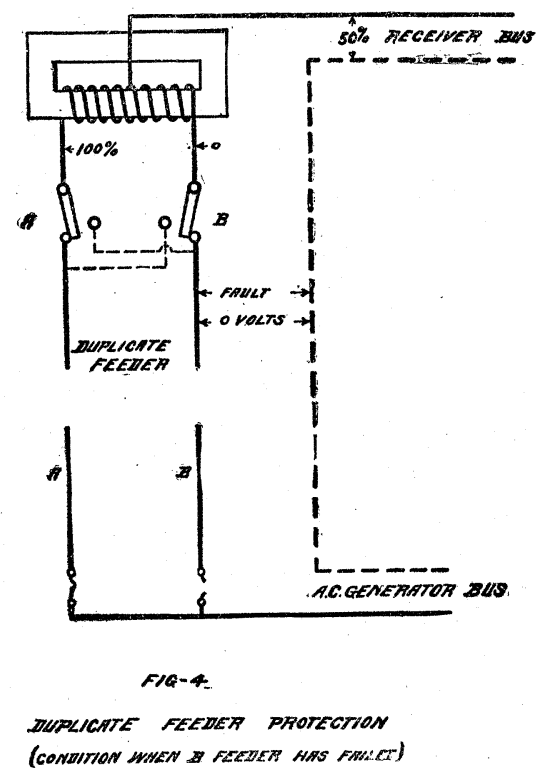

the particular feeder ana ine other on the mean voltage of the two feeders. Referring to Fig 5, the coils A and B are excited from the feeders $\mathrm{A}$ and $\mathrm{B}$ while coils $\mathrm{C} \mathrm{C}$ are connected as shown from the common terminal of $\mathrm{A}$ and $\mathrm{c}$ to the opposite polarity of the system. Normally, the two windings on each transformer magnetize in the same direction; but in case of a short-circuit on one of the feeders, while the current in $\mathrm{c} \mathrm{c}$ will continue in the same direction, the current in the coil connected to the faulty feeder will reverse, and the opposition of the two windings will send a flux through the middle leg on which is wound the secondary coil connected to the release-coil of the feeder switch. 
As the essential feature of the Andrews system is the use of the special choke-coils. a few words on the design of these coils is necessary. Under normal conditions there is no flux in the iron core and the only losses are those due to the ohmic resistance of the coils. When a fault occurs, the ampere-turns of the two coils will be unbalanced and the resultant of the two will produce a magnetic flux which reacting on the windings will tend to prevent the unbalancing of the currents. For complete protection, it will be seen that this flux must be sufficient to induce the full

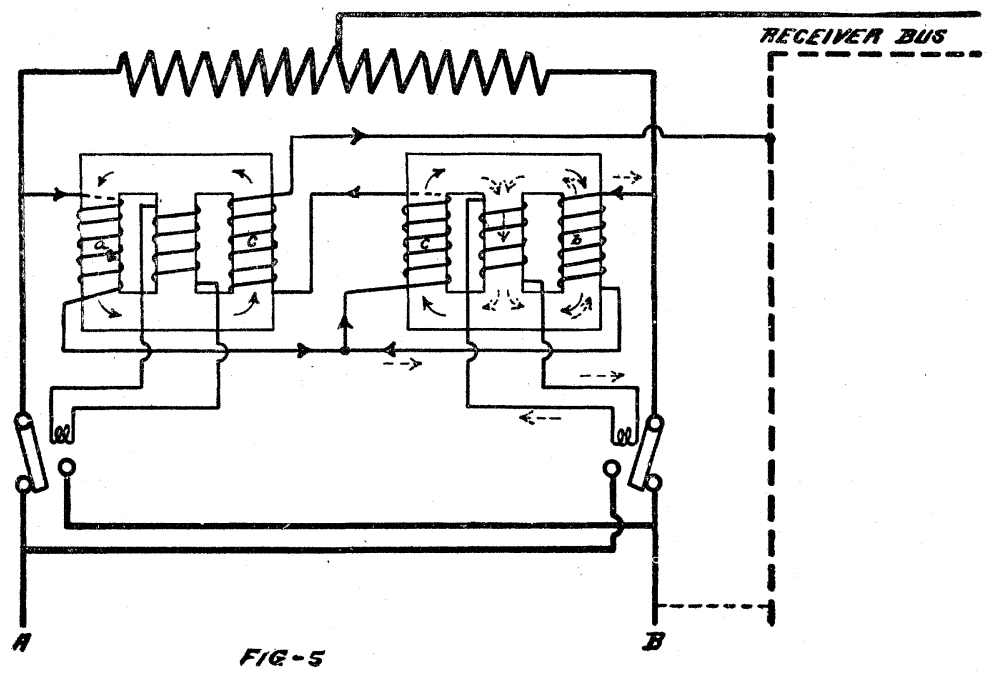

\section{(BRONEN-LINE ARAONS SHON GONOITION}

WNEN DO REEDER WAS FAILED.)

line pressure in the coils, but under this condition there is no objection to the iron being highly saturated; and consequently by careful attention to the design a very compact choke-coil can be produced.

To sum up, the use of choke-coils in this manner will introduce a small extra copper loss into the system, in return for which it will confer complete freedom from trouble due to short-circuits on the line by its direct action of choking becls the short-circuit current. 